\title{
Cost-Effectiveness of Trastuzumab With or Without Chemotherapy as Adjuvant Therapy in HER2-Positive Elderly Breast Cancer Patients: A Randomized, Open-Label Clinical Trial, the RESPECT Trial
}

\author{
Yuki Takumoto $^{1,2}$ (D) Takeru Shiroiwa ${ }^{1} \cdot$ Kojiro Shimozuma $^{3} \cdot$ Hiroji Iwata $^{4} \cdot$ Masato Takahashi $^{5} \cdot$ Shinichi Baba $^{6} \cdot$ \\ Kokoro Kobayashi ${ }^{7}$. Yasuhiro Hagiwara ${ }^{8}$. Takuya Kawahara ${ }^{9} \cdot$ Yukari Uemura $^{10}$. Hirofumi Mukai ${ }^{11}$. Naruto Taira ${ }^{12}$. \\ Masataka Sawaki $^{4}$
}

Accepted: 1 February 2022 / Published online: 1 March 2022

(c) The Author(s) 2022

\begin{abstract}
Background and Objective Trastuzumab is a standard care as adjuvant chemotherapy (AdjCT) for patients with human epidermal growth factor receptor 2 (HER2)-positive primary breast cancer (BC) in Japan. However, no reports have evaluated its economics for patients with HER2-positive BC over 70 years of age. The objective of this study was to evaluate the cost-effectiveness of HER2-targeted trastuzumab + chemotherapy in Japan, comparing it with trastuzumab monotherapy. Methods A three-state-partitioned survival model was developed to evaluate the cost-effectiveness of trastuzumab + chemotherapy versus trastuzumab monotherapy for AdjCT in elderly patients with HER2-positive BC. We derived the efficacy data, utilities, and costs of both arms from individual patient data in the RESPECT trial (NCT01104935) and published studies. The costs and quality-adjusted life years (QALYs) were discounted at $2 \%$ per annum using a payer perspective. The respective cost estimates were reported in 2019 Japanese Yen (JPY) or US dollars (US\$). The primary outcome was the incremental cost-effectiveness ratio (ICER). We assured robustness with deterministic and probabilistic sensitivity analyses. Results The cost per patient for trastuzumab + chemotherapy was JPY 14.6 million (US\$137,000), and their QALYs were 9.308, compared with JPY 14.2 million (US\$131,000) and 9.101, respectively, for trastuzumab monotherapy. The ICER of trastuzumab + chemotherapy versus trastuzumab monotherapy was JPY 2.7 milllion/QALY (US\$17,200/QALY). The ICER for trastuzumab with chemotherapy varied from "Dominant" to "Dominated" in one-way sensitivity analysis.

Conclusions The base-case analysis suggests that AdjCT with trastuzumab + chemotherapy is likely to be a cost-effective choice for patients with HER2-positive BC aged 70 years or older. However, the sensitivity analysis suggested uncertainty regarding the cost-effectiveness of trastuzumab + chemotherapy.
\end{abstract}

\section{Introduction}

Breast cancer (BC) remains the most common cancer among Japanese women, with an estimated 106,583 diagnoses in 2018. The proportion of patients over 70 years, who were the target population in this study, was $34.1 \%$ of all patients [1]. BC treatment is cardinally selected based on assessments of patient states and prognostic factors. The Japanese Breast Cancer Society's clinical practice guidelines 2018 and National Comprehensive Cancer Network guidelines recommend surgical resection in the early stage of cancer (stages $0-2$ ). In addition, neoadjuvant or adjuvant chemotherapy

Yuki Takumoto

Takumoto.y.aa@gmail.com

Extended author information available on the last page of the article

\section{Key Points}

We examined the cost-effectiveness of trastuzumab + chemotherapy as adjuvant chemotherapy (AdjCT) in patients with human epidermal growth factor receptor 2 (HER2)-positive breast cancer (BC) aged over 70 years in Japan, comparing it with trastuzumab monotherapy using the three-state partitioned survival model.

The base-case analysis suggests that AdjCT with trastuzumab + chemotherapy is likely to be a cost-effective choice for patients with HER2-positive BC aged 70 years or older.

In contrast, this result was uncertain because the incremental cost-effectiveness ratios and quality-adjusted life years of trastuzumab + chemotherapy mainly depended on the number of events and long-term efficacy in both arms. 
(AdjCT) may be administered, depending on the size of the tumor [2,3].

Medicines used in adjuvant treatment for $\mathrm{BC}$ are classified according to the presence of hormone receptors and human epidermal growth factor receptor type 2 (HER2), and include hormonal therapy, molecular targeted drugs, and other approaches. Several studies have reported the efficacy of trastuzumab as an adjuvant with chemotherapy, showing improved disease outcomes in patient with HER2-positive primary BC [4-6]. This combination regimen has been the standard treatment for HER2-positive primary BC in Japan [7-9].

Additionally, the increase in medical expenditure for cancer treatment is a major social issue in developed countries. Therefore, it is essential to not only assess treatment efficacy and safety, but also the cost of various treatment modalities that are used to treat cancer. Having access to such information is also essential [10]. So far, health economic evaluation has not been used for political decision-making in Japan. However, examining the health economic aspects of expensive medicines used in oncology, including BC, would significantly help to clarify the medical resource allocation guidelines. The benefits of trastuzumab in AdjCT, which we discuss in this study, have been evaluated from the economical aspect, and many studies have reported it to be cost-effective [11-19]. However, several safety risks have been reported for trastuzumab with chemotherapy, and its use may be avoided in elderly patients with BC [20-22]. Few studies have examined its effectiveness in AdjCT in patients aged over 70 years. Moreover, we have yet to uncover how the improved efficacy and safety from adding chemotherapy to trastuzumab monotherapy will affect the costeffectiveness of this regimen. The objective of this study was to examine the cost-effectiveness of trastuzumab + chemotherapy as an AdjCT of patients with HER2-positive primary BC over 70 years in Japan, comparing it with trastuzumab monotherapy. The purpose of this study was one of the secondary endpoints in the RESPECT trial, a randomized, open-label clinical trial. The RESPECT trial was registered with ClinicalTrials.gov (identifier: NCT01104935) on 6 November 2009 [23].

\section{Methods}

\subsection{Patient Population and Clinical Trial}

The base-case population modeled in this study was Japanese women with HER2-positive primary BC with a median patient age at treatment initiation of 75 years in the RESPECT trial. We chose trastuzumab + chemotherapy as the comparison arm $(\mathrm{H}+\mathrm{CT}$ arm $)$ and trastuzumab monotherapy as the control arm ( $\mathrm{H}$ arm) to achieve the study objective.
Briefly, the main inclusion criteria of the RESPECT trial were invasive BC diagnosed as HER2-positive; stage I, IIA, IIB, or IIIA cancer; and left ventricular ejection fraction above $55 \%$. The full analysis set (FAS) of this trial was made up of patients who initiated the intervention during the treatment period. Extrapolation parameters for cost utility analysis in this study were calculated from FAS results in the RESPECT trial. The patient background of FAS and patient flow diagram in the RESPECT trial are described in the Online Supplementary Material (OSM) Table 1 and Fig. 1. In the RESPECT trial, trastuzumab in both arms comprised a loading dose of $8 \mathrm{mg} / \mathrm{kg}$ and a maintenance dose of $6 \mathrm{mg} / \mathrm{kg}$ every 3 weeks for 1 year. Chemotherapy in the $\mathrm{H}+\mathrm{CT}$ arm involved one of the prescribed regimens (paclitaxel, docetaxel, doxorubicin + cyclophosphamide, epirubicin, cyclophosphamide + methotrexate +5 -fluorouracil, docetaxel+ cyclophosphamide, and docetaxel + carboplatin) for each patient for up to 24 weeks.

\subsection{Model Structure and Design}

A partitioned-survival model was developed to evaluate the cost-effectiveness of the $\mathrm{H}+\mathrm{CT}$ arm versus the $\mathrm{H}$ arm for AdjCT in older patients with HER2-positive primary BC. A partitioned survival model is a common modeling structure in the field of oncology health technology assessment process and is recommended by the NICE Decision Support Unit [24]. Three health states were created in a partitioned survival model, namely stable disease (SD), progressive disease (PD), and death (Fig. 1). In this model, the proportion of patients in each health state in each cycle was estimated by calculating the area under the curve per cycle from the disease-free survival (DFS) and overall survival (OS) curves for each study arm.

Therefore, all patients in this model entry were either on trastuzumab with chemotherapy or trastuzumab only as the SD in the first month. After the second month, the percentage of patients in each health state varied according to the percentage of DFS and OS for each treatment arm. The duration of treatment for trastuzumab and chemotherapy was assumed to follow the protocol rules of the RESPECT trial. Specifically, trastuzumab was administered for 1 year, and other chemotherapies were administered for between 12 and 24 weeks, depending on the regimen. We calculated the total effectiveness and total costs for each study arm over the time horizon of the model. The effectiveness in this study was quantified as quality-adjusted life years (QALYs). These were used to estimate the incremental cost-effectiveness ratios (ICERs).

We analyzed from the payer's perspective, including the direct medical cost involved in a patient's treatment. The cycle length was 30 days ( 1 month) to capture the impact on both arms in detail. The base-case analysis period was 
Table 1 Input utility parameters

\begin{tabular}{llllllll}
\hline Status & Period & H+CT & & & & Reference \\
& & Mean & STD & & Mean & STD & \\
\hline Stable disease & Baselines & 0.819 & 0.164 & 0.781 & 0.153 & RESPECT trial [23] \\
& 1-2 months & 0.782 & 0.171 & 0.829 & 0.158 & RESPECT trial [23] \\
& 3-12 months & 0.818 & 0.164 & 0.841 & 0.152 & RESPECT trial [23] \\
& 13-36 months & 0.808 & 0.159 & 0.846 & 0.172 & RESPECT trial [23] \\
Progressive disease & After 48 months & 0.715 & & & & & Lloyd et al. [34] \\
\hline
\end{tabular}

$H+C T$ trastuzumab with chemotherapy, $H$ trastuzumab monotherapy

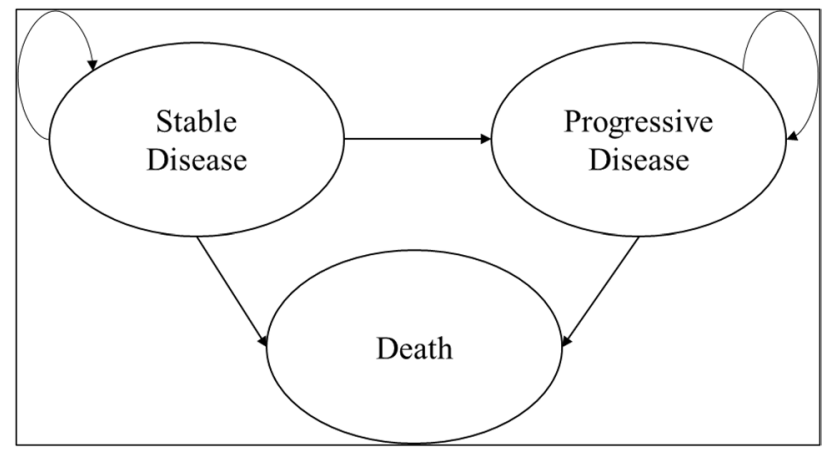

Fig. 1 Partitioned survival model with three health states

30 years to follow patients throughout their lifetime. All costs and QALYs in the base case were discounted at $2 \%$ per annum (in accordance with the guidelines for cost-effectiveness evaluation in Japan) [25]. The partitioned survival models in this study were created using Microsoft Excel (Microsoft Corporation, 365 MSO, 64 bit), and all extrapolation parameters were analyzed using SAS version 9.4 (SAS Institute, Cary, NC, USA) and R version 3.6.3. In reporting the results of this study, we adhered to ISPOR's good reporting practice guidelines for health economic evaluations (CHEERS checklist) [26].

\subsection{Efficacy}

We derived the clinical efficacy (DFS and OS durations) of each arm using individual patient data of FAS in the RESPECT trial. Specifically, we estimated parametric curves to model the probability of OS and DFS during and after the RESPECT trial using individual patient data and model functions. Model functions for DFS and OS parametric curves were selected from exponential, Weibull, Gompertz, log-normal, log-logistic, and generalized gamma distributions based on assessments of curve visualization and goodness of fit given by the Akaike Information Criterion (AIC) value and Bayesian information criterion (BIC)
[27]. This process of curve estimation with these model functions is the recommended option in the NICE Decision Support Unit [24].

We also confirmed the clinical and biological plausibility of the curve based on the consistency of the estimated DFS and OS parametric curves and natural history in Japan [28, 29]. We estimated a parametric curve for OS using data from the RESPECT trial with up to 5 years of follow-up because the median follow-up of the trial was 4.1 years (range 0.3-8.0 years), and most patients in the trial were censored at the time of follow-up. However, for the curve selection of visualization, we also referred to the Kaplan-Meier curve generated based on the data of the whole period. The upper limit for the estimation of the OS curve was set using life tables for the general population in Japan, based on the "Statistics Japan Report, 2018" [30]. The survival of a few patients with long-term follow-up in the trial was considered to be useful in estimating the survival curve. The monthly proportion of health states in SD was calculated from the selected DFS parametric curve, and the monthly proportion of health states in PD was calculated from the difference between the DFS parametric curve and selected OS parametric curve.

\subsection{Health-Related Utilities}

Health-related utility values were derived from individual patient data of the FAS population in the RESPECT trial based on EuroQol 5-Dimension 3-Level (EQ-5D-3L) questionnaires developed by the EuroQol Group task force [31]. The EQ-5D-3L index used five levels of severity in the five dimensions (mobility, self-care, usual activities, pain/discomfort, and anxiety/depression) [32]. We calculated the health-related utility value based on the response of individual patients and utility value set in Japan [33]. The EQ5D-3L questionnaires in this study were administered at baseline, 2 months, 1 year, and 3 years. All 232 patients included in this study $(\mathrm{H}+\mathrm{CT}$ arm: $113, \mathrm{H}$ arm: 119) were available at baseline of the RESPECT trial (OSM Table 2a). 
Due to limited patient data, we extrapolated utility values from the study by Lloyd et al. as health-related utility values in the long-term SD and PD states [34] (Table 1).

\subsection{Costs}

We derived cost parameters from the receipt data of individual patients in the RESPECT trial as monthly direct medical costs based on receipts and out of hospital prescription data.

The direct cost was defined as "all costs due to resource use that are entirely attributable to the use of a medical intervention or disease treatment" based on receipt data and outof-hospital prescription data [35]. In the RESPECT trial, 30 patients ( $\mathrm{H}+\mathrm{CT}$ arm: $14, \mathrm{H}$ arm: 16$)$ had been randomly selected for receipt data collection ("Cost population," OSM Table 2b). Specifically, we calculated the costs in the base case for the SD and PD states from the start of follow-up to 2 years after the beginning of the RESPECT trial (Table 2).

To account for variability in individual patient costs in the RESPECT trial, we calculated the costs for each arm at 6,12 , and 24 months. Because no substantial change in cost was expected unless there was disease progression occurred, we assumed that the cost at month 25 and beyond in the SD state would be the same as at month 24 . We estimated the cost of the PD state using patient data of both arms for the PD state in the RESPECT trial because very few patients in that trial reached the PD state. All costs were presented in 2019 Japanese yen (JPY) and converted to US dollars (US\$, JPY 1 = US\$107). In the COST population, we examined the ICER calculations and utility value, which shows the trends of the population. The results of the utility value in the COST population were similar to those of the overall population. Furthermore, when the patient background of the cost population was compared with the overall population, no significant bias in patient background was identified
(OSM Table 3). Therefore, we examined the effects of various parameters using sensitivity analysis.

\subsection{Sensitivity Analysis}

We conducted probabilistic sensitivity analysis (PSA) to estimate the uncertainty in this analysis [36]. DFS, OS, utility values, and costs were calculated with the bootstrapping method using individual patient data from the RESPECT trial. In bootstrapping, we repeated the process 2,000 times, randomly selecting the same number of subjects as in the RESPECT trial from individual patient data and calculating the ICERs. The model function to estimate DFS and OS parametric curves were fixed to those of the base-case analysis, since we examined parameter (sampling) uncertainty in this PSA.

We performed the one-way sensitivity analysis (OWSA) to consider variations in the ICERs when the range of parameters changed independently. The cost parameter was varied by up to $\pm 20 \%$ and the utility parameter was varied by up to $\pm 5 \%$. The range of variation was set from the $95 \%$ confidence interval (CI) for each parameter. The OWSA was performed using time horizons of 10 or 15 years and a discount rate of $3 \%$. In addition, we also performed two scenario analyses. We assumed no difference in OS between the two arms or in OS or quality of life (QOL) after 36 months.

\section{Results}

\subsection{Efficacy Results}

The Gompertz and generalized gamma distributions were, respectively, found to be the best fits for the DFS and OS data in the RESPECT trial (Fig. 2). These distributions were

Table 2 Input cost parameters (Japanese Yen (JPY)/month)

\begin{tabular}{|c|c|c|c|c|c|c|}
\hline \multirow[t]{2}{*}{ Status } & \multirow[t]{2}{*}{ Period } & \multicolumn{2}{|l|}{$\mathrm{H}+\mathrm{CT}$} & \multicolumn{2}{|l|}{$\mathrm{H}$} & \multirow[t]{2}{*}{ Reference } \\
\hline & & Mean & STD & Mean & STD & \\
\hline \multirow[t]{4}{*}{ Stable disease } & $0-6$ months & 245,447 & 189,797 & 220,061 & 102,582 & RESPECT trial [23] \\
\hline & $7-12$ months & 215,146 & 119,305 & 202,290 & 98,640 & RESPECT trial [23] \\
\hline & 13-24 months & 111,379 & 102,890 & 60,190 & 81,863 & RESPECT trial [23] \\
\hline & After 25 months & 28,963 & & & & Estimation $^{\mathrm{a}}$ \\
\hline Progressive disease & & 166,893 & & & & Estimation $^{\mathrm{b}}$ \\
\hline
\end{tabular}

$H+C T$ trastuzumab with chemotherapy, $H$ trastuzumab monotherapy

${ }^{a}$ Assuming that the cost at month 25 and beyond in stable disease would continue to be the cost at month 24 in the RESPECT trial

${ }^{\mathrm{b}}$ Estimated patient data of both arms for the progressive disease state in the RESPECT trial 
Table 3 Total cost, total QALYs, and ICER for each regimen

\begin{tabular}{llll}
\hline Result & $\mathrm{H}+\mathrm{CT}$ & $\mathrm{H}$ & Difference \\
\hline Total cost (JPY) & $14,752,181$ & $14,196,642$ & 555,539 \\
$\quad$ Stable disease & $7,862,234$ & $6,625,437$ & $1,236,798$ \\
$\quad$ Progressive disease & $6,889,947$ & $7,571,205$ & $-681,259$ \\
Total QALYs & 9.308 & 9.101 & 0.206 \\
$\quad$ Stable disease & 6.848 & 6.398 & 0.450 \\
$\quad$ Progressive disease & 2.460 & 2.703 & -0.243 \\
ICER (JPY/QALY) & $2,691,739$ & - & - \\
\hline
\end{tabular}

$Q A L Y$ quality-adjusted life year, ICER incremental cost-effectiveness ratio, $H+C T$ trastuzumab with chemotherapy, $H$ trastuzumab monotherapy

selected from AIC, BIC as well as estimated curve visualization results (OSM Table 4 and OSM Fig. 2).

\subsection{Base-Case Analysis}

Over a lifetime horizon of 30 years, the total cost per patient for trastuzumab + chemotherapy was calculated as JPY 14.6 million (US\$137,000), compared with JPY 14.2 million (US\$132,000) for trastuzumab monotherapy, and the total QALYs were 9.308 and 9.101, respectively (Table 3). The incremental cost of trastuzumab with chemotherapy per patient was JPY 0.56 million (US\$5000), and the incremental QALYs of trastuzumab with chemotherapy was 0.206 . As a result, the ICERs of trastuzumab with chemotherapy versus trastuzumab monotherapy was JPY 2.7 million/QALY (US\$25,200/QALY).

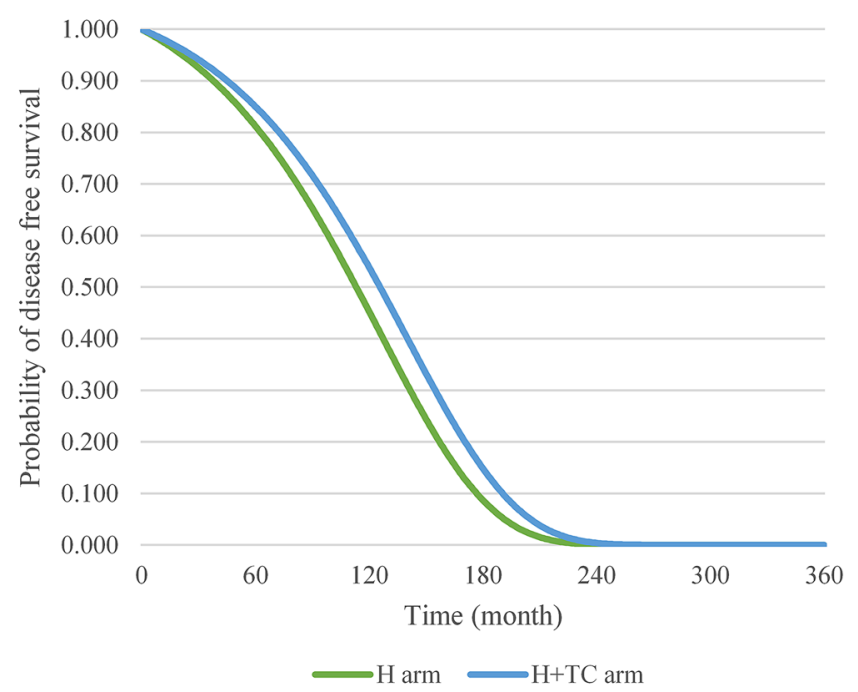

\subsection{Probability Sensitivity Analysis}

The results from the PSA (2000 bootstrapping simulations) showed that the mean ICER of trastuzumab with chemotherapy versus trastuzumab monotherapy was JPY 2.9 million/ QALY, (US\$26,900/QALY) (Fig. 3). The cost-effectiveness acceptability curves showed that at a willingness-to-pay threshold of JPY 5.0 million/QALY, the probability of being cost-effective was $51.75 \%$ for trastuzumab with chemotherapy (Fig. 4). However, when we excluded the ICERs with negative values, the probability that the ICER would be less than JPY 5.0 million/QALY was $62.2 \%$.

\subsection{One-Way Sensitivity Analysis}

Table 4 shows the results of the seven OWSAs with the effects on the base-case ICER of trastuzumab + chemotherapy against trastuzumab monotherapy. Scenarios with independent variations in cost and QALYs of SD and PD had a large impact on the ICERs. The range of ICERs in cost scenario (Scenarios 1 and 2) was between dominant and JPY 10.3 million/QALY (between dominant and US\$96,400/ QALY). The range of ICER in utility value scenario (Scenarios 3 and 4) was between JPY 1.01 million/QALY and dominated (between US\$9,460/QALY and dominated).

Additionally, the analysis assuming no difference in OS between the two groups (Scenario 8) and assuming no difference in OS and utility value after 36 months (Scenario 9) resulted in an increase in the total cost and total QALYs in the $\mathrm{H}$ arm (difference in total cost from $\mathrm{H}+\mathrm{CT}=\mathrm{JPY}$ 0.39 million, difference in total QALY from $\mathrm{H}+\mathrm{CT}=$ $0.073 \sim 0.107)$.

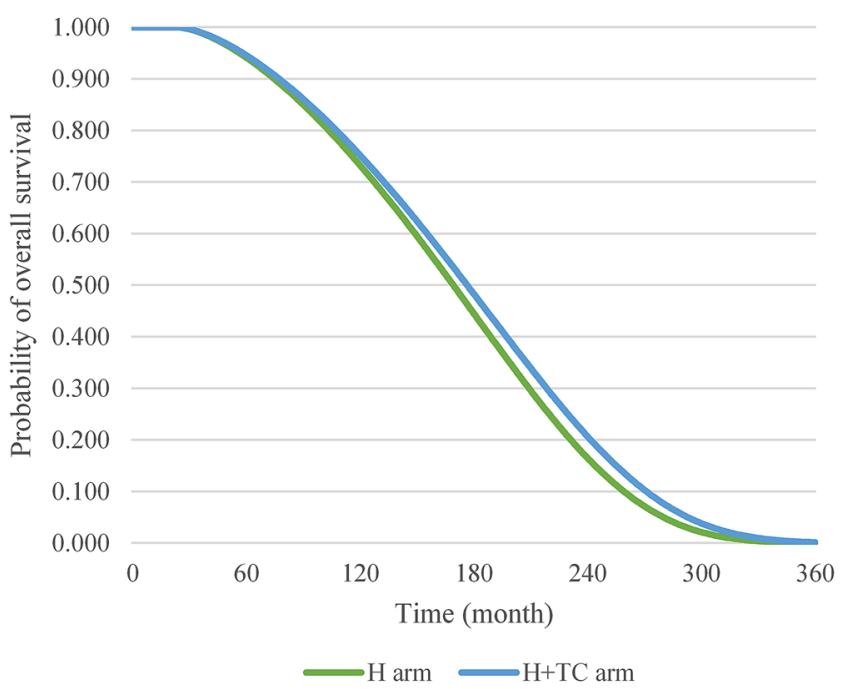

Fig. 2 Estimated disease-free survival and overall survival curves. $H+C T$ trastuzumab with chemotherapy, $H$ trastuzumab monotherapy 
Table 4 One-way sensitivity analysis

\begin{tabular}{|c|c|c|c|}
\hline \multirow[t]{3}{*}{ Scenario } & \multirow[t]{3}{*}{ Basis for setting } & \multicolumn{2}{|l|}{ ICER } \\
\hline & & \multicolumn{2}{|l|}{ (JPY/QALY) } \\
\hline & & Lower or base & Upper \\
\hline 1-A: Cost of stable disease in $\mathrm{H}+\mathrm{CT}$ arm & $\pm 20 \%$ & Dominant & $10,310,676$ \\
\hline 1-B: Cost of stable disease in $\mathrm{H}$ arm & $\pm 20 \%$ & $9,112,151$ & Dominant \\
\hline 2-A: Cost of progressive disease in $\mathrm{H}+\mathrm{CT}$ arm & $\pm 20 \%$ & Dominant & $9,368,476$ \\
\hline 2-B: Cost of progressive disease in $\mathrm{H}$ arm & $\pm 20 \%$ & $10,028,653$ & Dominant \\
\hline 3-A: Utility value of stable disease in $\mathrm{H}+\mathrm{CT}$ arm & $\pm 5 \%$ & Dominated & $1,012,299$ \\
\hline 3-B: Utility value of stable disease in $\mathrm{H}$ arm & $\pm 5 \%$ & $1,055,537$ & Dominated \\
\hline 4-A: Utility value of progressive disease in $\mathrm{H}+\mathrm{CT}$ arm & $\pm 5 \%$ & $6,661,475$ & $1,686,633$ \\
\hline 4-B: Utility value of progressive disease in $\mathrm{H}$ arm & $\pm 5 \%$ & $1,626,578$ & $7,798,701$ \\
\hline 5: Using a X-year time horizon & 10 year & Dominated & - \\
\hline 6: Using a X-year time horizon & 15 year & $11,860,467$ & - \\
\hline 7: Using X\% discount rate for both costs and QALYs & $3 \%$ & $3,237,786$ & - \\
\hline 8: The OS curve of both arms is equal ${ }^{\mathrm{a}}$ & - & $3,011,252$ & - \\
\hline $\begin{array}{l}\text { 9: The OS curve and utility value after } 36 \text { months of both arms are } \\
\text { equal }^{\text {a }}\end{array}$ & - & $4,433,041$ & - \\
\hline
\end{tabular}

$Q A L Y$ quality-adjusted life year, ICER incremental cost-effectiveness ratio, $H+C T$ trastuzumab with chemotherapy, $H$ trastuzumab monotherapy ${ }^{\mathrm{a}}$ Control arm was $\mathrm{H}+\mathrm{CT}$ arm

\section{Discussion}

We conducted a cost-effectiveness analysis of the AdjCT of trastuzumab with chemotherapy against trastuzumab monotherapy for patients with HER2-positive primary BC aged over 70 years. The efficacy of both arms was demonstrated in a randomized, open-label clinical trial, known as the RESPECT trial. No characteristic bias was observed in the patient background between the two arms in the RESPECT study. We compared the $\mathrm{H}+\mathrm{CT}$ arm with the $\mathrm{H}$ arm for adjuvant therapy for patients with HER2-positive BC aged 70 years or older. The ICER of the $\mathrm{H}+\mathrm{CT}$ arm over the
Fig. 3 Scatter plot of probabilistic sensitivity analysis results. $W T P$ willingness to pay, $Q A L Y$ quality-adjusted life year

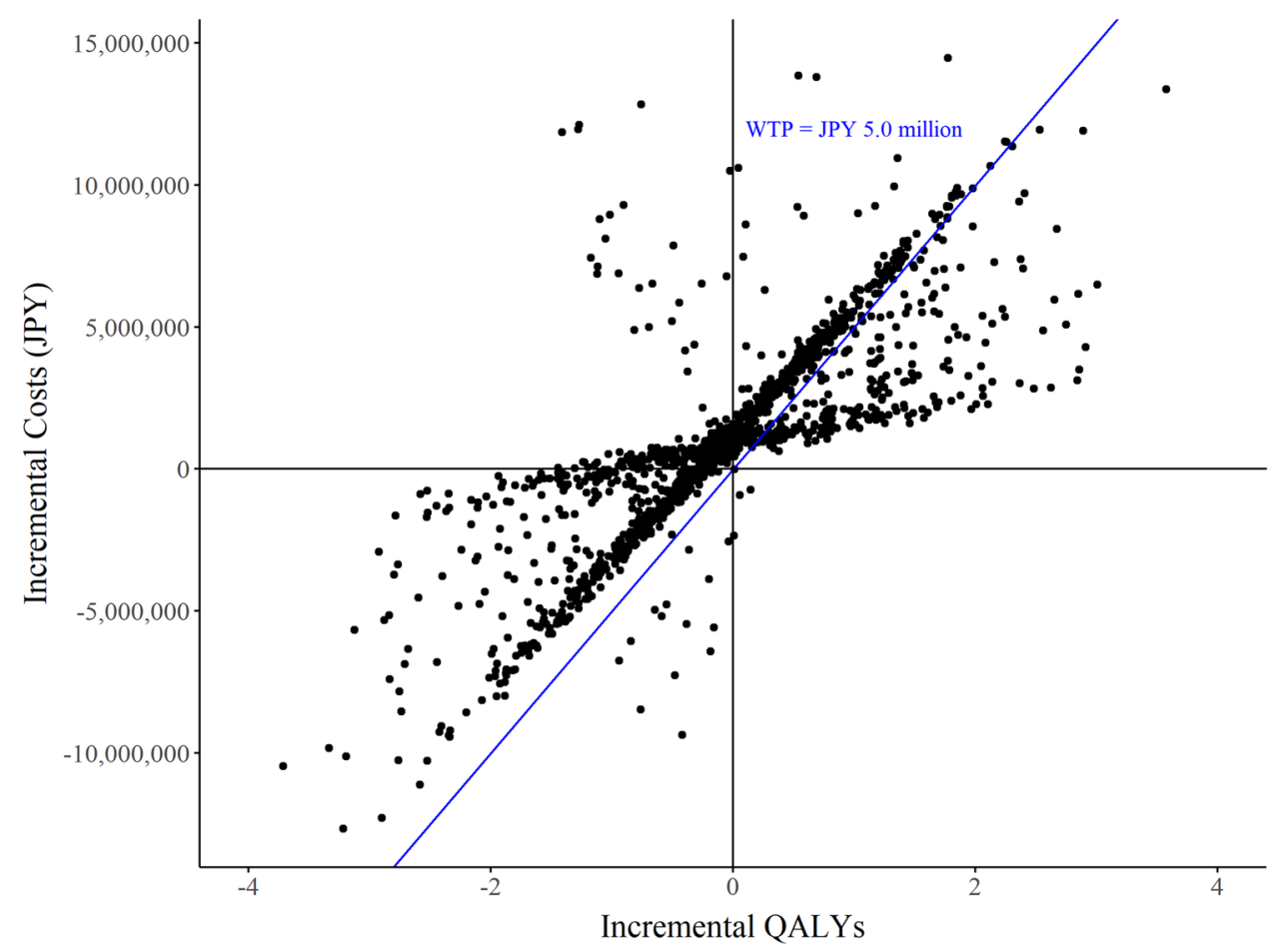


Fig. 4 Cost-effectiveness acceptability curves. $Q A L Y$ quality-adjusted life year

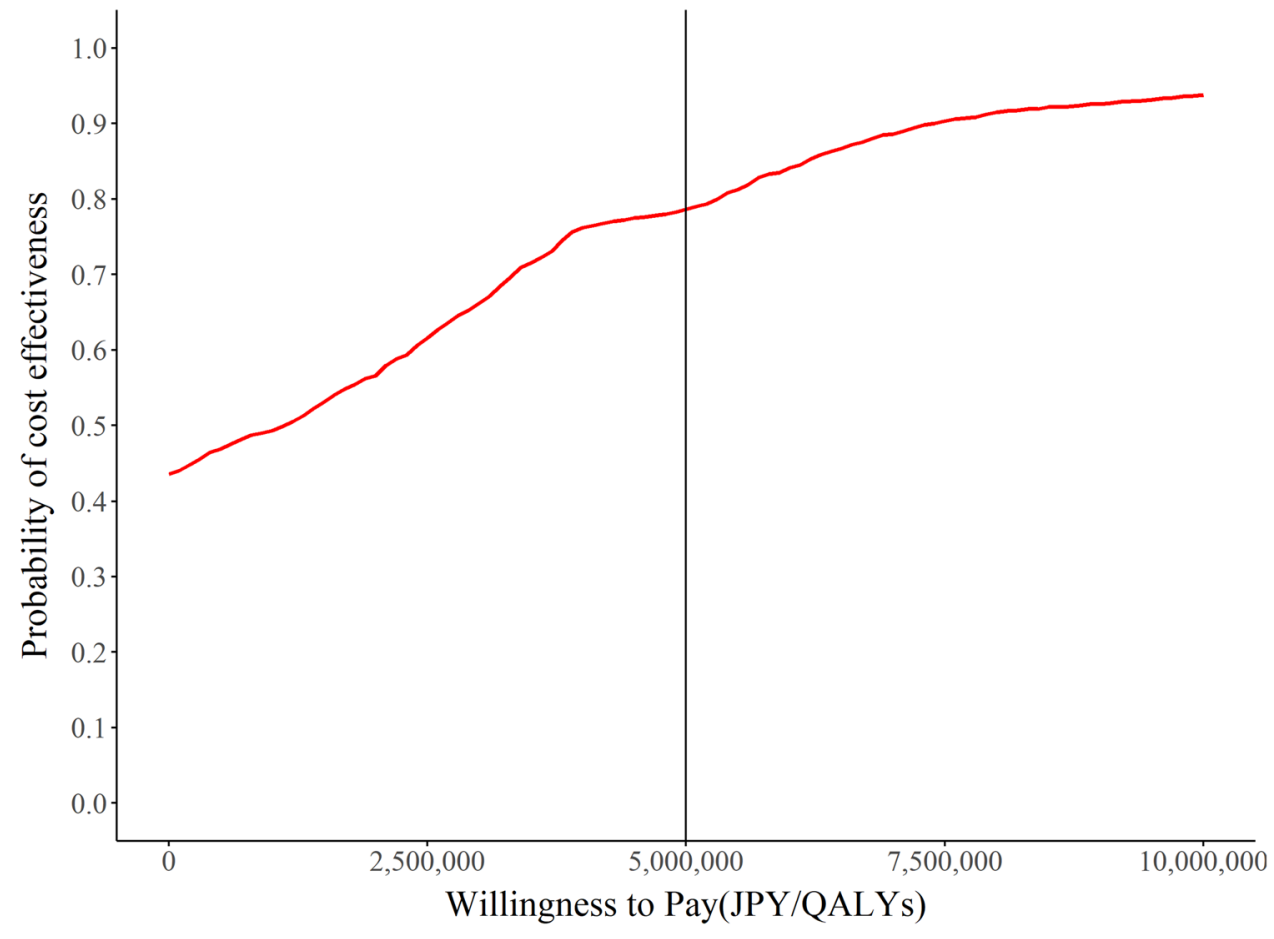

H arm was JPY 2.7 milllion/QALY (US\$25,200/QALY). Although there is no clear threshold in Japan for the superiority of ICER-based cost-effectiveness analysis results, considering the study by Shiroiwa et al., we could assume that the $\mathrm{H}+\mathrm{CT}$ arm with an ICER of less than JPY 5 million/ QALY was cost-effective based on the results of the base case [37]. However, the PSA results showed an extensive range of variability in the ICERs due to changes in parameters. Additionally, Moreover, because the HR of the OS in the RESPECT trial was 1.07 (95\% CI, 0.36 to 3.19) and no significant difference in OS was observed, we set up Scenarios 8 and 9 to examine the degree of uncertainty in OS and long-term utility. We found that the total cost and total QALYs for the $\mathrm{H}$ arm were higher than for the H+CT arm. Furthermore, the clinically meaningful HRQoL deterioration rate showed significant differences 1 year (19\% vs. 38\%; $P$ value $=0.009$ ). The results of Scenario 9 suggest that even though the rate of conversion to PD with greater disutility was higher in the $\mathrm{H}$ arm, the $\mathrm{H}$ arm with less disutility during SD had a more substantial impact on total QALYs. In other words, the disutility of trastuzumab + chemotherapy as AdjCT in elderly patients with BC might have a greater impact on total QALYs than the utility of trastuzumab + chemotherapy with a slight extension of DFS. Thus, the greater disutility of adding chemotherapy to trastuzumab may have a greater impact on total QALYs than the utility gained by a small extension of DFS. These results suggest a high uncertainty in the ICER based on long-term estimates of efficacy parameters using the current evidence.
There are three main reasons for this uncertainty. First, the probability of occurrence was low in the RESPECT trial due to the characteristics of BC. The 10-year survival rate for BC in Japan is approximately $80 \%$ for all patients and over $90 \%$ for patients with stage 0 or $1 \mathrm{BC}$. In the RESPECT trial, more than $80 \%$ of patients had stage 1 or 2 BC. Most subjects did not experience events, such as stage progression or death, during the average 4-year follow-up period. However, long-term estimation of efficacy may have an increasing impact on the ICER and is a source of expanding uncertainty of the ICER. To minimize the uncertainty in the long-term estimation of efficacy, we used OS for 5 years after the start of this model. We also evaluated the curve concerning the curve visualization and natural history of DFS, OS, and the general Japanese population to select an appropriate model function. This suggests that further follow-up trials for evaluating the long-term efficacy in both arms are necessary to perform more accurate analyses.

Second, the difference in survival between the two arms is very small. The loss of OS in the $\mathrm{H}$ arm versus the $\mathrm{H}+\mathrm{CT}$ arm over 3 years of follow-up was less than 1 month in the RESPECT trial, and the difference in incremental QALYs between the two arms in this analysis was only 0.206 in a lifetime (30 years). Therefore, the short analysis period affected the ICER. When the time horizon was 15 years, the ICER of the $\mathrm{H}+\mathrm{CT}$ arm over the $\mathrm{H}$ arm was more than JPY 10 million/QALY. Therefore, considering that the average age of the subjects at the start of the RESPECT trial was 74 years and the life expectancy of women in Japan is 87.5 years (2019), it is suggested that it is uncertain whether 
$\mathrm{H}+\mathrm{CT}$ is cost-effective for patients with a low life expectancy [38].

Thirdly, the difference in the total cost and QALYs between the two arms is very small. In the base case, the difference in total cost between the two arms is approximately JPY 0.5 million (US\$4670), and the difference in total QALY between the two arms is only 0.206 . This difference is even smaller in Scenarios 8 and 9, where the OS and longterm utility value differences are eliminated. Therefore, even if the variation in the cost and utility parameters in OWSA is small, the impact on the ICER is large, and the ICER will fluctuate significantly. This suggests that the economic advantages and disadvantages of the two arms are small.

This study has three limitations. First, the RESPECT study has limited patient data for accurate estimation of direct medical costs. In this study, we collected patient receipt data in the RESPECT trial to calculate the cost parameters so as to increase internal validity. Since the cost parameters collected in the RESPECT trial had a large variability, we were concerned about the impact on the ICER. However the patients used to estimate the cost parameters were randomly selected and their backgrounds do not differ significantly from the overall population, we believe that the cost parameters can ensure a certain degree of representativeness in the RESPECT trial. On the other hand, the variability is not small, and the maximum standard deviation in the cost parameter is approximately JPY 189,700 (US\$1770). In fact, from Scenarios 1 and 2, the ICER of the $\mathrm{H}+\mathrm{CT}$ arm varied from dominant to more than JPY 10 million $(\$ 93,500)$. Therefore, for future analysis, a sample size of at least 150 participants per arm should be used.

Second, the long-term utility value is uncertain. Utility values in the RESPECT trial were measured using the EQ5D-3L up to 36 months. In this trial, the utility value after 37 months was analyzed by extrapolating the utility value of metastatic BC in previous UK studies because there are no Japanese studies on such parameters [34]. The target population for the outfitted utility values included patients with metastatic $\mathrm{BC}$ but not those aged over 70 years, which was the target population for this study. The study in Japan has also reported that age is strongly correlated with QOL values, suggesting that utility values decrease with age [39]. Furthermore, we conducted our analysis using utility values of metastatic $\mathrm{BC}$, which is more advanced in stage than our target disease, i.e., postoperative BC. This is because we can conduct the most conservative analysis possible without overestimating the effect of each arm.

Finally, since the RESPECT trial is an open-label trial, there are concerns that it may affect the determination of DFS and the measurement of utility value.

Based on these analyses, because the ICER of the $\mathrm{H}+\mathrm{CT}$ arm against the $\mathrm{H}$ arm in the base-case analysis was below JPY 5.0 million/QALY, the economic advantage of $\mathrm{H}+\mathrm{CT}$ therapy over $\mathrm{H}$ therapy is not necessarily guaranteed. The reason for this is the uncertainty of the long-term parameters of efficacy, cost, and utility value. To resolve these uncertainties, it will be necessary to collect additional information on the long-term prognosis and associated health-care costs and utilities after AdjCT for BC in elderly patients. In addition, the small difference in the total cost and total QALY between the two arms may also be a factor that increases the uncertainty. Furthermore, our sensitivity analysis showed that $\mathrm{H}$ therapy might have similar resource consumption and QALY gain as $\mathrm{H}+\mathrm{CT}$ therapy. Therefore, the benefit of $\mathrm{H}$ therapy as AdjCT for BC in elderly patients with deteriorating systemic conditions should be further investigated.

\section{Conclusion}

The ICER for trastuzumab with chemotherapy as adjuvant chemotherapy for patients with HER2-positive BC aged 70 years or older in Japan was below JPY 5.0 million/QALY. On the other hand, it was suggested that additional investigation of some parameters with the uncertainty that could have a large impact on ICER is needed. Finally, our findings suggest that the resources consumed and QALYs gained by both treatments may be comparable.

Supplementary Information The online version contains supplementary material available at https://doi.org/10.1007/s40261-022-01124-y.

Acknowledgements This study was supported by the data center in the Public Health Research Foundation.

\section{Declarations}

Funding This study was funded by the Comprehensive Support Project for Oncology Research (CSPOR) of the Public Health Research Foundation, Japan. All decisions concerning the planning, implementation, and publication of this study were made by the executive committee of this study. The corporate and individual sponsors of this study are listed on the CSPOR website (http://www.csp.or.jp/cspor/kyousan_e.html).

Conflict of interest HI has a potential conflict of interest with Chugai Pharmaceutical Co. Ltd, Pfizer Inc, and Sanofi S.A. MT has a potential conflict of interest with Chugai Pharmaceutical Co. Ltd.

Availability of data and material Our data are available only on the CSPOR data center.

Code availability Not applicable.

Ethics approval This study was one of the secondary endpoints in the RESPECT trial, which was a randomized, open-label clinical trial. The study protocol of the RESPECT trial conformed with the guidelines of the Declaration of Helsinki and the Ethical Guidelines for Clinical Research of Japan's Ministry of Health, Labor, and Welfare. It was approved by an independent ethics committee for each participating site. All participants provided written informed consent. 
Consent to participate We used data from the RESPECT trial, which obtained informed consent from the respondents under those conditions.

\section{Consent for publication Not applicable.}

Author contributions YT and TS were involved in the conception and design of the study. All authors contributed to the analysis or interpretation of data for this work, contributed in drafting or revising it critically for important intellectual content, and approved the final version.

Open Access This article is licensed under a Creative Commons Attribution-NonCommercial 4.0 International License, which permits any non-commercial use, sharing, adaptation, distribution and reproduction in any medium or format, as long as you give appropriate credit to the original author(s) and the source, provide a link to the Creative Commons licence, and indicate if changes were made. The images or other third party material in this article are included in the article's Creative Commons licence, unless indicated otherwise in a credit line to the material. If material is not included in the article's Creative Commons licence and your intended use is not permitted by statutory regulation or exceeds the permitted use, you will need to obtain permission directly from the copyright holder. To view a copy of this licence, visit http://creativecommons.org/licenses/by-nc/4.0/.

\section{References}

1. National Cancer Registry in Japan (2016-2018). Cancer Statistics. Cancer Information Service, National Cancer Center, Japan (National Cancer Registry, Ministry of Health, Labour and Welfare). https://ganjoho.jp/reg_stat/statistics/data/dl/excel/cancer_ incidenceNCR(2016-2018).xlsof subordinate document. Accessed 29 July 2021.

2. Iwata H, Saji S, Ikeda M, et al. The Japanese Breast Cancer Society Clinical Practice Guidelines, 2018 edition: the tool for shared decision making between doctor and patient. Breast Cancer. 2020;27(1):1-3. https://doi.org/10.1007/s12282-01901021-x (Epub 2019 Nov 22. Erratum in: Breast Cancer. 2021;28(4):987).

3. Gradishar WJ, Anderson BO, Abraham J, et al. Breast cancer, Version 3.2020, NCCN clinical practice guidelines in oncology. J Natl Compr Canc Netw. 2020;18(4):452-78.

4. Smith I, Procter M, Gelber RD, et al. 2-year follow-up of trastuzumab after adjuvant chemotherapy in HER2-positive breast cancer: a randomised controlled trial. Lancet. 2007;369(9555):29-36.

5. Perez EA, Romond EH, Suman VJ, et al. Four-year follow-up of trastuzumab plus adjuvant chemotherapy for operable human epidermal growth factor receptor 2-positive breast cancer: joint analysis of data from NCCTG N9831 and NSABP B-31. J Clin Oncol. 2011;29(25):3366-73.

6. Slamon D, Eiermann W, Robert N, et al. Breast Cancer International Research Group. Adjuvant trastuzumab in HER2-positive breast cancer. N Engl J Med. 2011;365(14):1273-83.

7. Romond EH, Perez EA, Bryant J, et al. Trastuzumab plus adjuvant chemotherapy for operable HER2-positive breast cancer. N Engl J Med. 2005;353:1673-84.

8. Piccart-Gebhart MJ, Procter M, Leyland-Jones B, et al. Trastuzumab after adjuvant chemotherapy in HER2-positive breast cancer. N Engl J Med. 2005;353:1659-72.

9. Garrison LP Jr, Babigumira J, Tournier C, et al. Cost-effectiveness analysis of pertuzumab with trastuzumab and chemotherapy compared to trastuzumab and chemotherapy in the adjuvant treatment of HER2-positive breast cancer in the United States. Value Health. 2019;22(4):408-15.

10. Gold MR, Siegel JE, Russell LB, Weinstein MC. Cost effectiveness in health and medicine. New York: Oxford University Press; 1996.

11. Hajjar A, Ergun MA, Alagoz O, Rampurwala M. Cost-effectiveness of adjuvant paclitaxel and trastuzumab for early-stage nodenegative, HER2-positive breast cancer. PLoS ONE. 2019;14(6): $\mathrm{e} 0217778$.

12. Kunst N, Wang SY, Hood A, et al. Cost-effectiveness of neoadjuvant-adjuvant treatment strategies for women with ERBB2 (HER2)-positive breast cancer. JAMA Netw Open. 2020;3(11): e2027074.

13. Garrison LP Jr, Lubeck D, Lalla D, Paton V, Dueck A, Perez EA. Cost-effectiveness analysis of trastuzumab in the adjuvant setting for treatment of HER2-positive breast cancer. Cancer. 2007;110(3):489-98. https://doi.org/10.1002/cncr.22806 (PMID: 17592827).

14. Lidgren M, Jönsson B, Rehnberg C, Willking N, Bergh J. Costeffectiveness of HER2 testing and 1-year adjuvant trastuzumab therapy for early breast cancer. Ann Oncol. 2008;19(3):487-95. https://doi.org/10.1093/annonc/mdm488 (Epub 2007 Dec 6 PMID: 18065409).

15. Liberato NL, Marchetti M, Barosi G. Cost effectiveness of adjuvant trastuzumab in human epidermal growth factor receptor 2-positive breast cancer. J Clin Oncol. 2007;25(6):625-33. https:// doi.org/10.1200/JCO.2006.06.4220 (Erratum in: J Clin Oncol. 2007;25(25):4030. PMID: 17308267).

16. Kurian AW, Thompson RN, Gaw AF, Arai S, Ortiz R, Garber AM. A cost-effectiveness analysis of adjuvant trastuzumab regimens in early HER2/neu-positive breast cancer. J Clin Oncol. 2007;25(6):634-41. https://doi.org/10.1200/JCO.2006.06.3081 (PMID: 17308268).

17. Macedo A, Monteiro I, Andrade S, Cirrincione A, Ray J. Custoefectividade de trastuzumab no tratamento de doentes com cancro da mama em estádios iniciais em Portugal [Cost-effectiveness of trastuzumab in the treatment of early stages breast cancer patients, in Portugal]. Acta Med Port. 2010;23(3):475-82 (Portuguese. Epub 2010 Jun 14. PMID: 20654267).

18. Ansaripour A, Uyl-de Groot CA, Redekop WK. Adjuvant trastuzumab therapy for early HER2-positive breast cancer in Iran: a cost-effectiveness and scenario analysis for an optimal treatment strategy. Pharmacoeconomics. 2018;36(1):91-103. https://doi.org/ 10.1007/s40273-017-0557-6 (Erratum in: Pharmacoeconomics. 2018 Feb 23. PMID: 28795341; PMCID: PMC5775392).

19. Norum J, Olsen JA, Wist EA, Lønning PE. Trastuzumab in adjuvant breast cancer therapy. A model based cost-effectiveness analysis. Acta Oncol. 2007;46(2):153-64. https://doi.org/10.1080/ 02841860601096841 (PMID: 17453363).

20. Pinder MC, Duan Z, Goodwin JS, et al. Congestive heart failure in older women treated with adjuvant anthracycline chemotherapy for breast cancer. J Clin Oncol. 2007;25:3808-15.

21. Du XL, Xia R, Liu CC, et al. Cardiac toxicity associated with anthracycline-containing chemotherapy in older women with breast cancer. Cancer. 2009;115:5296-308.

22. Patt DA, Duan Z, Fang S, et al. Acute myeloid leukemia after adjuvant breast cancer therapy in older women: understanding risk. J Clin Oncol. 2007;25:3871-6.

23. Sawaki M, Taira N, Uemura Y, RESPECT study group, et al. Randomized controlled trial of trastuzumab with or without chemotherapy for HER2-positive early breast cancer in older patients. J Clin Oncol. 2020;38(32):3743-52.

24. Woods B, Sideris E, Palmer S, et al. NICE DSU technical support document 19. Partitioned survival analysis for decision modelling in health care: a critical review. NICE Decision Support Unit, 2017. http://nicedsu.org.uk/wp-content/uploads/2017/06/Parti 
tioned-Survival-Analysis-final-report.pdf of subordinate document. Accessed 29 July 2021.

25. Center for Outcomes Research and Economic Evaluation for Health. Official guideline for cost-effectiveness evaluation. Guideline for cost-effectiveness evaluation in Japan (second edition) [English]. https://c2h.niph.go.jp/tools/guideline/guideline_en.pdf of subordinate document. Accessed 29 July 2021.

26. Husereau D, Drummond M, Petrou S, ISPOR Health Economic Evaluation Publication Guidelines-CHEERS Good Reporting Practices Task Force, et al. Consolidated Health Economic Evaluation Reporting Standards (CHEERS) — explanation and elaboration: a report of the ISPOR Health Economic Evaluation Publication Guidelines Good Reporting Practices Task Force. Value Health. 2013;16(2):231-50.

27. National Institute of Health and Care Excellence Guide to the methods of technology appraisal. 2013. https://www.nice.org.uk/ process/pmg9/resources/guide-to-the-methods-of-technologyappraisal-2013-pdf-2007975843781 of subordinate document. Accessed 29 July 2021.

28. Statistic Japan report in 2018, Table 2-20 Data is available by 5 years of age. http://www.stat.go.jp/data/nihon/02.html of subordinate document. Accessed 29 July 2021.

29. Source for natural mortality rates in Japan in 2016-2017. https:// www.mhlw.go.jp/toukei/list/81-1.html of subordinate document. Accessed 29 July 2021

30. Statistic Japan report in 2018, Table 2-20 Data is available by 5 years of age http://www.stat.go.jp/data/nihon/02.html. Accessed 29 July 2021.
31. Rabin R, de Charro F. EQ-5D: a measure of health status from the EuroQol Group. Ann Med. 2001;33(5):337-43.

32. Szende A, Oppe M, Devlin NJ. EQ-5D value sets: inventory, comparative review and user guide. Dordrecht: Springer; 2007.

33. Tsuchiya A, Ikeda S, Ikegami N, et al. Estimating an EQ-5D population value set: the case of Japan. Health Econ. 2002;11(4):341-53

34. Lloyd A, Nafees B, Narewska J, et al. Health state utilities for metastatic breast cancer. Br J Cancer. 2006;95(6):683-90.

35. Direct costs. In: Kirch W, editor. Encyclopedia of public health. Springer, Dordrecht. https://doi.org/10.1007/978-1-4020-5614-7 799.

36. Briggs AH, Wonderling DE, Mooney CZ. Pulling cost-effectiveness analysis up by its bootstraps: a non-parametric approach to confidence interval estimation. Health Econ. 1997;6(4):327-40.

37. Shiroiwa T, Igarashi A, Fukuda T, et al. WTP for a QALY and health states: more money for severer health states? Cost Eff Resour Alloc. 2013;1(11):22.

38. Government Statistical Office (e-Stat), abridged life table in 2019(female). https://www.e-stat.go.jp of subordinate document. Accessed 29 July 2021 (Japanese).

39. Shiroiwa T, Fukuda T, Ikeda S, et al. Japanese population norms for preference-based measures: EQ-5D-3L, EQ-5D-5L, and SF-6D. Qual Life Res. 2016;25(3):707-19.

\section{Authors and Affiliations}

\section{Yuki Takumoto ${ }^{1,2}$ (1) $\cdot$ Takeru Shiroiwa $^{1} \cdot$ Kojiro Shimozuma $^{3} \cdot$ Hiroji Iwata $^{4} \cdot$ Masato Takahashi $^{5} \cdot$ Shinichi Baba $^{6}$. Kokoro Kobayashi ${ }^{7}$. Yasuhiro Hagiwara ${ }^{8} \cdot$ Takuya Kawahara $^{9} \cdot$ Yukari Uemura $^{10}$. Hirofumi Mukai ${ }^{11}$ - Naruto Taira ${ }^{12}$. Masataka Sawaki ${ }^{4}$}

1 Department of Health and Welfare Services, Center for Outcomes Research and Economic Evaluation for Health, National Institute of Public Health, 2-3-6, Wako, Saitama 351-0104, Japan

2 Meiji Pharmaceutical University, Tokyo, Japan

3 Department of Biomedical Sciences, College of Life Sciences, Ritsumeikan University, Kusatsu, Japan

4 Department of Breast Oncology, Aichi Cancer Center Hospital, Nagoya, Japan

5 Department of Breast Surgery, NHO Hokkaido Cancer Center, Sapporo, Japan

6 Department of Surgery, Sagara Hospital, Kagoshima, Japan

7 Department of Medical Oncology, The Cancer Institute Hospital of the Japanese Foundation for Cancer Research, Tokyo, Japan
8 Department of Biostatistics, Graduate School of Medicine, The University of Tokyo, Tokyo, Japan

9 Biostatistics Division, Clinical Research Promotion Center, The University of Tokyo Hospital, Tokyo, Japan

10 Biostatistics Section, Department of Data Science, Center for Clinical Sciences, National Center for Global Health and Medicine, Tokyo, Japan

11 Department of Breast and Medical Oncology, National Cancer Center Hospital East, Kashiwa, Japan

12 Department of Breast and Endocrine Surgery, Okayama University Hospital, Okayama, Japan 